INSERT COIN:

PAY-TO-WIN AND

THE LOOT BOX

DILEMMA

\section{NATHAN RESTER}

Nathan Rester is a student at the University of Mississippi School of Law in Oxford, Mississippi

\section{I. “WOULD YOU KINDLY BUY A LOOT BOX?”}

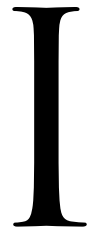

$\mathrm{t}$ is one of gaming's most iconic sounds, immediately recognizable to millions around the world. Mario the Plumber leaps upward, a single hand outstretched toward a dangling gold disc floating enticingly in the air. And as Mario snatches up yet another coin, the player hears a brief but unmistakable jingle. This sound effect is composed of only two notes, but it is instantly familiar to anyone who has ever picked up a copy of Super Mario Bros. or engaged with its legion of successors and spin-offs. It is a sound familiar to grandparents and grandchildren alike, to gamers who play once a month, and those who log multiple hours a day. This seemingly innocuous jingle is paramount to the Mario franchise and to the current state of the gaming industry as a whole.

While it is difficult to gauge the exact correlation between this pithy sound effect and the ultimate success of the Mario franchise, musical cues such as the coin jingle are designed to "motivate the player to perform the actions that connect the sequence of the game experience by rewarding successful behaviour [sic] and punish failure," noting that the telltale ring of collecting coins in Mario has become "universal in games that involve collecting coins," and that "success in [games] is similarly reinforced by a musical "reward." "These "micro-rewards" formed the essential basis of Nintendo's success. While games like pinball "offered basically no correlation between what you do (pull a plunger) and the 'reward' of a hundred buzzers," games like Super Mario Bros. and Donkey Kong offered a player-based reward schedule, gratifying players as they interacted with the game while simultaneously encouraging future interactions: "like a slot machine . . . this was a formula for a stratospherically addicting game, one in which either your skill or your luck may make all the difference next game. That is, until you were out of quarters."

While the video game industry is now decades past the peak of Mario's success, the essential formula created and fostered by games like Super Mario Bros. has remained intact: a labyrinth of periodic, gamer-driven rewards designed to beckon the player onward, all in the service of picking one last quarter out of their pocket. The two-note jingle remains, in essence if not in practice, in virtually every video game released since the original Super Mario Bros. Because that player-based system of reward continues to succeed, the same compulsively driven behavior continues to dominate the modern gaming industry: "Instant gratification is no longer a benefit; it is a requirement and millennials - those born between 1980 to 2000 - have seldom experienced anything else." 3 This is a valid point pertinent not only to games, but to the way entertainment is consumed in general. In the same way that weekly television installments have been replaced with all-day Netflix binges, so too have video games been streamlined to correlate with modern tastes and attitudes. This represents not only a shift in what types of games are played, but in how those games are delivered to players. The introduction of loot boxes into modern gaming begs the question of whether the innocuous cheerleader of a telltale jingle has evolved into a sinister and predatory siren song. The more pressing question is whether such evolution marks the transition of video gaming from a harmless pastime into a harmful and potentially illegal manifestation of gambling.
Keywords: video gaming, Battlefront, loot box, addiction, gambling

DOI: $10.1089 / \mathrm{glr} 2.2019 .2346$ (C) 2019 Mary Ann Liebert, Inc.

${ }^{1}$ Zach Whalen, Play Along - An Approach to Videogame Music, 4(1) Game Studies 214 (2004).
${ }^{2}$ Jeff Ryan, Super Mario: How Nintendo Conquered America (2011).

${ }^{3}$ Desirée Martinelli, Skin Gambling: Have We Found the Millennial Goldmine or Imminent Trouble?, 21(8) GAMING L. Rev. 557 (2017). 
The explosion of video games can be in no small measure attributed to the availability of the technology necessary to deliver these games electronically combined with the addicting quality of the games. While the days of tossing quarters into a bottomless pit at the arcade are over, the same essential game can now be carried out in the comfort of the player's own home. Statistically, many players find themselves dependent on or even addicted to video games. In a large study of 7,000 gamers, "nearly $12 \%$ of participants complied with three or more modified criteria for addiction and were therefore considered to be pathological gamers." ${ }^{44}$ Video games are infamously habit-forming, and even gamers who are not technically "addicted" to their hobby still show a strong predisposition toward such a condition. According to a study referenced by Zaheer Hussain, "Fortyone percent of people who play online video games admitted that they played computer games as an escape from the real world. The researchers classified seven percent of these gamers as 'dependent,' [using] video games to modify their moods."

As video games have expanded their financial and cultural territory, psychologists have posited several theories about their addictive nature. The dominating theory is responsible for the addicting nature of video games: "dopamine is a neurotransmitter that has a number of functions in the brain and is involved in mood, sleep, voluntary movements, and situations of reward. It is this latter function that draws our attention." ${ }^{, 6}$ In order to understand the psychological consequences of video game play, a group of British researchers designed a study to determine the physiological link between video games and dopamine. Observing that "most drugs of addiction produce pleasure by increasing the amount of dopamine in the brain," the study utilized a form of brain imaging called positron emission tomography or PET to determine whether playing video games increased the amount of dopamine released by the brain. Their research found "a massive increase in the amount of

${ }^{4}$ Sabine M. Grüsser et al., Excessive Computer Game Playing: Evidence for Addiction and Aggression?, 10(2) Cyber Psychol. \& Behav. 291 (2006).

${ }^{5}$ Zaheer Hussain and Mark D. Griffiths, Excessive Use of Massively Multi-Player Online Role-Playing Games: A Pilot Study, 7(4) Int'l J. Mental Health and AdDiction 563 (2009).

${ }^{6}$ Scott Rigby and Richard M. Ryan, Glued to Games: How Video Games Draw Us In AND Hold Us Spellbound (2011) dopamine released in the brain was indeed observed during video game play, in particular in areas thought to control reward and learning." 7

It is this physiological response that keeps customers playing and, indeed, paying to play. There seems to be no question in the minds of researchers and concerned parents whether video games are habit-forming. From a consumer protection standpoint, it would appear a natural element of product development for video game developers and publishers to capitalize on the seemingly symbiotic relationship between gamer and game for profit.

"Pay-to-win" systems have created undeniably significant sources of revenue for their developers, who are naturally incentivized to monetize their intellectual property. Games such as League of Legends, Dota 2, Hearthstone, Overwatch, and Counter-Strike: Global Offensive use in-game roulettes called "loot boxes" as a massive source of their revenue stream by allowing players to use real-world money to unlock undisclosed in-game perks of varying value to the player: "Players can play the game for free, but purchase mystery boxes that may or may not contain in-game items they want," and the final effect is essentially "like a slot machine, but where you put in real dollars to try to win virtual gear instead of money."

For many games (e.g., Dota 2, League of Legends, and Hearthstone), loot or mystery boxes are their sole source of revenue. ${ }^{9}$ Players get "hooked" on a free product but ultimately feel compelled to spend actual money on loot boxes. For several years, free-to-play games represented an experiment, largely insulated from the traditional gaming industry. However, the industry has adopted pay-to-win systems and loot boxes previously implemented by smaller developers working on independent and mobile games. While many free-to-play games still use these wage-based mechanics, full-priced games have also begun introducing loot box elements.

\footnotetext{
${ }^{7}$ C. Shawn Green and Daphne Bavelier, The Cognitive Neuroscience of Video Games, in Digital Media: Transformations in Human Communication 211 (Paul Messaris and Lee Humphreys, eds., 2006)

${ }^{8}$ Chaim Gartenberg, China's New Law Forces Dota, League of Legends, and Other Games to Reveal Odds of Scoring Good Loot, THE VERGE (May 2, 2017), https:// www.theverge.com/2017/5/2/15517962/china-new-law-dota-league-of-legendsodds-loot-box-random. 
In these cases, loot box systems allow gamers to circumvent initial levels of tedious gameplay by unlocking rewards more quickly. In single-player games, players are encouraged to "skip playing the actual game, by awarding them loot and [gear] they'd have to acquire manually elsewhere," and this has led many commentators to suggest that "loot boxes are being built into the core of these games, replacing systems that worked just fine before, or that were better than what they're being replaced with," essentially altering games to operate around the concept of the loot crates rather than the other way around. ${ }^{10}$ While this phenomenon is relatively new, the introduction of loot boxes into traditional full-price games has fundamentally changed the nature of what is considered a "full" game.

Conventional console and PC games operate much like any other form of "fixed" media like a movie or an album - a flat fee paid at the moment of purchase would guarantee that the consumer "owned" the product in the same sense that every other consumer did. At this point, the transaction between publisher and consumer would traditionally conclude. However, by inserting systems and mechanisms designed for free-to-play games into games that are charged at full price, developers are essentially creating a secondary market within the games themselves that allows players to enhance the initial experience of the base game via a series of microtransactions: while "cosmetic crates may still be relatively uncontroversial . . . other games attempting to rake in that same level of cash are drastically altering core components of their titles to do so, and it seems like it's only getting worse as time goes on."11 Loot boxes and their accompanying pay-towin systems do not simply act as "add-ons" for video games - they alter them at a chemical level.

For gamers, the introduction of free-to-play systems (once regulated to the fringes of mobile games, largely aimed at children and older adults) into full-

\footnotetext{
${ }^{10}$ Paul Tassi, How Shadow of War's Loot Boxes Cripple the Game, Even If You Can "Ignore" Them, Forbes (Nov. 22 2017), https://www.forbes.com/sites/insertcoin/ 2017/10/14/how-shadow-of-wars-loot-boxes-cripple-the-game-even-if-you-canignore-them/\#235af580397a.

${ }^{11} I d$.

${ }^{12}$ Stephen Totilo, The Strange, Scary, Fascinating, Exciting Future of Video Games, According to a Giant, KotaKu (June 20, 2012), https://kotaku.com/thestrange-scary-fascinating-exciting-future-of-vid-5919847.
}

priced games represents a significant shift for the industry. Developers and publishers alike seem all too aware of gamers' frustrations: "I want people to understand that what EA [Electronic Arts] employees and the people who create the games are working hard to do is pick our way through this transformation as best we can," says Peter Moore, chief operating officer (COO) of Electronic Arts. "We're a publicly-traded company. We have an obligation to, quite frankly, make money so we can re-invest money in making great games again." 12 Electronic Arts currently finds itself under the public's unflattering microscope; this situation is caused in no small part by the company's determination to utilize loot boxes and "make great games again." The controversy surrounding loot boxes could very well alter the foundations of the industry and pave the way for legislative action that would put the future of loot boxes in doubt.

Until November 2017, the controversy surrounding pay-to-win systems found itself more or less limited to the gaming community and found no real traction in the mainstream media. However, the release of Star Wars Battlefront II (published by Electronic Arts) sparked a massive backlash that was covered widely by conventional news outlets. The controversy centered around the game's inclusion of loot boxes and Electronic Arts' introduction in what is essentially a pay-to-win system in a multiplayer online game: "Players were able to buy in-game currency ('crystals') to use for in-game loot boxes, [which contain] a random smattering of items for use in the game," altering the game "in favor of people willing to spend more money, known among gaming fans as "pay to win." 13 Battlefront II "raised flags" because some consider the practice akin to gambling and the game is marketed heavily to children and most other competitive games do not offer "pay-towin" advantages. ${ }^{14}$ The attention drawn by the game devolves from the scale of the game and the seemingly exploitative nature of these loot boxes.

\footnotetext{
${ }^{13}$ Ben Gilbert, The New "Star Wars" Game Is Embroiled in Controversy, and Fans Are Furious-Here's What's Going On, Business Insider (Nov. 17 2017), https:// www.businessinsider.in/the-new-star-wars-game-is-embroiled-in-controversy-andfans-are-furious-heres-whats-going-on/articleshow/61695411.cms.

${ }^{14}$ Gene Park, How a Star Wars Video Game Faced Charges That It Was Promoting Gambling, WASH. Post (Nov. 18, 2017).
} 
Another common grievance centered around "gameplay systems that loot boxes are replacing by simply existing, and how games are designed to steer people toward loot box stores whether they want to be there or not." ${ }^{\prime 2}$ Loot boxes, while technically optional add-ons, are increasingly perceived as being part and parcel of a mandatory system of fees necessary to experience the "complete" experience of a particular game. The fallout from the controversial inclusion of loot boxes in Battlefront II was so seismic that it temporarily caused Electronic Arts' share price to drop $8.5 \%$, effectively eliminating $\$ 3.1$ billion in shareholder value. ${ }^{15}$ While Electronic Arts initially rescinded the implementation of loot boxes in light of the growing controversy, EA finance chief Blake Jorgensen later clarified that loot boxes would eventually be reinstated into the game "when we think it's ready." 16 Regardless of whether the video gaming community at large approves of loot boxes, Electronic Arts (and most likely other game publishers) seems determined to stay the course concerning its controversial implementation of pay-to-win systems in its releases.

\section{II. "YOUR PRINCESS IS IN ANOTHER CASTLE, SENATOR RANKER”}

Despite the obvious business and economic advantages of games based around the purchasing of loot boxes, publishers may face a darker tide than hyperbolic controversy drummed up by their consumer base. Even as Electronic Arts grapples with the dilemma of how to implement loot boxes in a nonintrusive manner, the controversy created by the release of Battlefront II continues to gain momentum. The debate around loot crates has entered the mainstream cultural conversation and a handful of elected officials have spoken adamantly about the issue, recognizing the seemingly predatory practices implemented in modern gaming and how these practices exploit children as a direct conduit to their parents' wallets. At the forefront of this debate is Washington State Senator Kevin Ranker. The father of two has attacked the usage of loot boxes as predatory, a clear abuse of an industry selling gambling to children under the innocent guise of video games: "It is unacceptable to be targeting our children with predatory gambling masked in a game with dancing bunnies or something."17

Ranker is not alone in his concern, even among elected officials. Chris Lee, a state representative from Hawaii, has publicly attempted to draft legislation regulating the sale of video games that feature loot boxes. Lee shares Ranker's sentiment, reflecting a growing concern among elected officials that these seemingly innocuous games are designed to target impressionable, financially oblivious children. "We know inside the development process for many of these games that there are clear and deliberate decisions being made to employ these kinds of mechanisms with the full knowledge of the effects they can have," Lee argues. "That's made obvious by the kinds of people who have been employed, including psychologists and other sorts of experts."18 Lee's thoughts on the issue have manifested into potentially game-changing actions. Four proposed bills recently introduced in the state of Hawaii "target exploitative monetization techniques in video games that some fear might psychologically condition players to become addicted to gambling," prohibiting the sale of any game featuring a "randomized reward system" that requires real currency to any potential buyer beneath the age of $21 .{ }^{19}$ If successful, the Hawaiian quartet of bills would mark the first time that loot boxes have been officially censored or sanctioned in the United States and would mark a massive paradigm shift in how these systems are implemented in the future.

However, there is no small amount of pushback against these measures. The Entertainment Software Rating Board (ESRB), a regulatory organization responsible for assigning ratings to video games to indicate
${ }^{15}$ Erik Kain, EA Shares Plummet After "Star Wars: Battlefront II" Loot Box Fiasco, Forbes (Nov. 29, 2017), https://www.forbes.com/sites/erikkain/2017/11/28/eashares-plummet-after-star-wars-battlefront-ii-loot-box-fiasco/\#4052cd666f37.

${ }^{16}$ Hope Corrigan, Battlefront II Misses Sales Expectations, Microtransactions Will Return "In the Next Few Months," IGN (Jan. 30, 2018), https://www.ign.com/ articles/2018/01/30/ea-blames-loot-box-controversy-for-star-wars-battlefront-iimissed-sales-expectations-microtransactions-will-return-in-the-next-few-months.
${ }^{17}$ Kyle Orland, The Legislative Fight over Loot Boxes Expands to Washington State, Ars Technica (Jan. 25, 2018), https://arstechnica.com/gaming/2018/01/thelegislative-fight-over-loot-boxes-expands-to-washington-state/.

${ }^{18}$ Kyle Orland, Meet the Legislator Trying to Do Something about Video Game Loot Boxes, Ars Technica (Dec. 16, 2017), https://arstechnica.com/gaming/2017/ 12 /meet-the-legislator-trying-to-do-something-about-video-game-loot-boxes/.

${ }^{19}$ Michael Brestovansky, Bills Target Video Games with Rewards for a Price, HawaII TRIBune-Herald (Feb. 12, 2018), https://www.hawaiitribune-herald.com/ 2018/02/12/hawaii-news/bills-target-video-games-with-rewards-for-a-price/. 
appropriate age groups and warning consumers about potentially offensive content, has stated that it does not consider loot boxes to be gambling: "While there's an element of chance in these mechanics, the player is always guaranteed to receive in-game content... We think of it as a similar principle to collectible card games ... [Sometimes] you'll end up with a pack of cards you already have." 20

As the primary regulatory body, the ESRB represents a powerful force in the gaming industry. The organization differentiates between real gambling and simulating - because the former is defined by its usage of real currency and the latter is defined by its usage of faux (in-game) currency, games that fall under the real gambling banner are given an "adults only" rating that precludes anyone under the age of 17 from purchasing them. ${ }^{21}$ Because of this, the ESRB's stance on the matter packs a significant amount of clout - their judgment reflects how games that promote and utilize loot boxes will be penalized/regulated in the immediate future.

And while bills like the quartet introduced in Hawaii may face an impossible uphill battle - both in literal court and in the court of public opinion - the United States would not be the first to place legal barriers between video game publishers and the emerging cash cow that is the loot box system. China has already passed legislation "that requires developers of video games that feature random loot boxes to reveal the odds of players receiving items," a rule has proven to have massive influence, with "multiple big-name companies revealing the drop rates for rare items in their loot boxes, many of them for the first time." ${ }^{, 2}$ While this regulation is a far cry from banning loot boxes outright or even sanctioning games that use them, the snowball effect it represents could signal an early apocalypse for loot boxes. Following a review of Star Wars: Battlefront II, FIFA 18, Overwatch, and Counter-Strike: Global Offensive, the
Belgian Gambling Commission found that only Battlefront II had avoided violation - due only to the fact that Electronic Arts removed the game's loot boxes prior to review. If FIFA 18, Overwatch, and Counter-Strike: Global Offensive do not remove their loot boxes, the publishers "risk a prison sentence of up to five years and a fine of up to 800,000 euros [and] when minors are involved, those punishments can be doubled." ${ }^{23}$ While it remains to be seen whether similar results will be reached in the United States, there is no question that loot boxes find themselves imperiled abroad.

In the United States, reservations expressed by government officials may be construed as political saber rattling, but there may yet be a realistic path toward the regulation of loot box systems. Martinelli notes that "the lack of regulation provides the perfect atmosphere for thirsty, tech-savvy entrepreneurs looking to capitalize on the craze" and that courts may very well find it necessary to regulate video games, "especially if a social policy concern such as underage gambling through esports and skins betting arises." ${ }^{24}$ Developers are simply taking advantage of a classic "millennial" issue - the need for instant gratification - and while boxes themselves have generated controversy, any potential regulation could be argued more as a preventative measure than a reactive one.

Construing games like Battlefront II as nursery casinos might sound hyperbolic, but expert David G. Schwartz, director of the University of Nevada Las Vegas's Center for Gaming Research, feels that the comparison is not too far-fetched: "For something to be considered gambling, the classic definition [is] risking something of value on an unknown outcome in hope of getting a payoff . . . With a loot box, it's pretty much that." 25 This begs the question: do Schwartz and Martinelli have their fingers on the pulse of a rapidly approaching change and
${ }^{20}$ Jason Schreier, ESRB Says It Doesn't See "Loot Boxes" as Gambling, Kotaku (Oct. 11, 2017), https://kotaku.com/esrb-says-it-doesnt-see-loot-boxes-asgambling-1819363091.

${ }^{21}$ ESRB Ratings Guide, EnTERTAINMEnt Software Rating Board, https://www.esrb. org/ratings/ratings_guide.aspx.

${ }^{22}$ Chaim Gartenberg, China's New Law Forces Dota, League of Legends, and Other Games to Reveal Odds of Scoring Good Loot, The Verge (May 2, 2017), https://www.theverge.com/2017/5/2/15517962/china-new-law-dota-league-oflegends-odds-loot-box-random.

\footnotetext{
${ }^{23}$ Wesley Yin-Poole, Now Belgium Declares Loot Boxes Gambling and Therefore Illegal, EuroGAMER.NeT (Apr. 4, 2018), https://www.eurogamer.net/articles/201804-25-now-belgium-declares-loot-boxes-gambling-and-therefore-illegal.

${ }^{24}$ Desirée Martinelli, Skin Gambling: Have We Found the Millennial Goldmine or Imminent Trouble?, 21(8) GAMING L. REv. 564 (2017).

${ }^{25}$ Zaid Jilani, Video Game "Loot Boxes" Are Like Gambling for Kids-and Lawmakers Are Circling, THE InTERCEPT (Dec. 8, 2017), https://theintercept.com/ 2017/12/08/video-games-loot-boxes-gambling-gaming-star-wars-battlefront-2/.
} 
subsequent tide of new legislation, or is the loot box issue just another footnote in the long and storied history of video game controversy?

That question can only be answered by an inquiry into gaming law itself. This inquiry necessitates an examination of the case law that contributes to the canon of debate regarding video games and their propensity for selling "gambling" in the guise of "dancing bunnies." When examining an activity in light of whether or not it constitutes gambling, three elements must be examined and satisfied: "consideration, prize and chance." ${ }^{26}$ By applying these three elements to loot boxes, the validity of arguments posited by concerned bystanders like Ranker and Lee are thrown into sharper focus.

In the case of the first element (consideration), the analysis is straightforward. According to the Second Restatement of Contracts, "In such typical bargains as the ordinary sale of goods each party gives up something of economic value, and the values exchanged are often roughly or exactly equivalent by standards independent of the particular bargain."27 This means that the consumer must pay something of value up front in order for there to be consideration - this can be money or any item or property that has some kind of inherent value. For anti-loot box opponents, a potential pitfall may arise here via the fact that many games allow players to "farm" for in-game currency that can be substituted for real world currency and subsequently used to purchase loot boxes. However, this is normally a secondary edition and is usually just a red herring meant to steer players decisively in the direction of paid content: "There could have been a million ways to use earned currency in [this game], but you are instead meant to spend $80 \%$ of your money on . . . loot boxes with randomized drops. This is, of course, meant to tempt you into buying [superior] loot boxes by simply putting you in the store., 28 While many games present in-game alternatives to

\footnotetext{
${ }^{26}$ State ex rel. Schillberg v. Safeway Stores, 75 Wash.2d 339, 340 (Wash. 1969).

${ }^{27}$ Restatement (SECOND) OF Contracts $\$ 79$ (2nd 1981).

${ }^{28}$ Paul Tassi, How Shadow of War's Loot Boxes Cripple the Game, Even If You Can "Ignore" Them, ForBes (Nov. 22, 2017), https://www.forbes.com/sites/insertcoin 2017/10/14/how-shadow-of-wars-loot-boxes-cripple-the-game-even-if-you-canignore-them/\#235af580397a
}

spending actual cash, these options are typically rote and designed specifically to drive players toward spending real money. It appears that loot boxes may in fact be the desired endgame of many developers, as many games are constructed from the ground up with micro-transactions in mind: in Battlefront II, even dedicated players are "still forced to use the game's loot crate system, which spits out bonuses entirely at random, to advance their characters and improve their loadouts," which "strips players of a sense of control" over the game. ${ }^{29}$

The second element (chance) also finds footing in the loot box debate. Gambling is largely defined by this element, by "staking or risking something of value upon the outcome of a contest of chance or a future contingent event not under the actor's control or influence, upon an agreement or understanding that he will receive something of value in the event of a certain outcome." ${ }^{30}$ This is the axis upon which an act of gambling turns, and Anthony Hawke, a representative for Māori Public Health Leadership (a public health organization that works to minimize gambling harm in New Zealand), asserts that the act of purchasing a loot box spins on the same essential fulcrum: “. . . when you're pushing the button on a slot machine there's no element of skill and knowledge. It's just randomized play. And that's what a loot box is . . . it's ingraining in our children an element of addiction from an early age." ${ }^{31}$ When purchasing a loot box, players know they will receive something of value (at least in the context of the game offering said loot box), but the exact contents are largely a mystery to players. Even the developers who offer loot boxes are not privy to the contents before being opened by the players, as the rewards are randomly generated by the game. This fulfills the second element and strengthens the argument for loot boxes as an illegal manifestation of gambling.

The final element (a prize) is also easily met. When purchasing loot boxes, players undoubtedly expect

\footnotetext{
${ }^{29}$ Owen S. Good, I Spent $\$ 90$ in Battlefront 2, and I Still Don't Have Any Control over My Characters, PolYGon (Nov. 16. 2017), https://www.polygon.com/2017/ 11/16/16658476/star-wars-battlefront-2-loot-crate-costs-analysis.

${ }^{30}$ Boardwalk Regency Corp. v. Attorney Gen. of New Jersey, 188 N.J. Super. 372, 373 (N.J. Super. Law Div. 1982)

${ }^{31}$ Adam Goodall, Are Loot Boxes the Slot Machines of Video Gaming?, THE SPINOFF (Dec. 19, 2017), https://thespinoff.co.nz/games/19-12-2017/lootboxes-slotmachines-video-gaming/.
} 
something of value. While the exact amount or exact value of the prize itself is insignificant, the final effect is paramount: as long as the prize itself has value, then the act is considered gambling.

While it could be argued that the "prizes" in loot boxes have no real-world value, this argument insinuates a complete misunderstanding of the medium. "The idea that the prizes in loot boxes have no real-world value imagines that the people who invest hours and hours into their video games would be unable to put a price on their progress or certain aspects of the game. They definitely could." 32 When purchasing a loot box, the player is gaining access to one or several items, which despite having no physical application outside of the digital construct created by the game, have value that directly affects the game and, in some cases, alters it in a meaningful way by offering in-game points, advantages, and weapons that would otherwise not be available. This enhances the core experience as provided by the base game, as proven by the incredibly lucrative market that the loot box mechanic has spawned. The items contained within the loot box have inherent value for someone who is already invested in the game to which those contents will later be applied. Therefore, it is reasonable to conclude that the loot boxes (or, more specifically, the items they contain) are of value, even if that value is only appreciated by an insular audience.

Arguments against the prohibition of loot boxes largely focus on precedent. Because video gaming is just another spoke in the wheel of gaming-related entertainment, proponents of pay-to-win systems point to previous iterations of gaming that failed to draw the intense scrutiny and debate that video games have. Loot boxes, they argue, are yet another incarnation of a form of entertainment that has endured unchallenged for decades. For example, the ESRB's argument stands upon the notion that loot boxes are essentially a digital equivalent to collecting and trading collectible cards. In the case of collectible trading cards (like baseball cards), courts have already ruled that buying packets of randomized cards (with a chance at snagging a rare card within the pack) does not constitute a form of gambling. In Major
League Baseball v. Price, the court stated that consumers who purchased baseball card booster packs were not participating in illegal gambling because they were technically receiving something of value with their purchase: by purchasing cards, consumers "[enter] into a bargain with the licensors and manufacturers whereby in return for payment the purchaser will receive a random assortment of [cards] ... This bargain delivers actual value to each party because the chance itself is of value regardless of whether or not the card purchaser later suffers a 'loss.",33 This ruling has remained unchallenged and stands as the definitive word on the issue of its day: whether or not boys and girls who collected cards were being coaxed into gambling.

Comparing loot boxes to trading cards is an easy assessment to make, as the similarities are apparent. Both involve the purchasing of self-contained packages, with the only distinction being that one package is a physical packet while the other is a digital crate. Both contain some sort of randomized item or allotment of randomized items. Both are purchased with the understanding that the item or items contained within may possess a valuation or range of valuations that differ depending on what type of loot box or booster pack the consumer has purchased. The only necessary distinction between physical playing cards and digital loot has already been noted: once purchased, the contents of a loot box cannot be returned, traded, or even discarded. Trading cards are, by name, meant to be traded from player to player based on the whims of the original purchaser. This subtracts an essential element of the trading card experience from the equation of loot boxes and draws attention to a fundamental problem with the ESRB's reasoning. A trading card holds a fundamental, inherent value all on its own: it can be traded, sold, and purchased secondhand. Trading cards, once purchased, open the doorway to a secondary market of trading card enthusiasts. Even if the initial consumer relinquishes his pursuit of baseball cards and essentially solidifies his collection, the collection itself still exists in physical form - that collection can later be sold or bartered away or appreciated as a standalone, untethered experience.
${ }^{32}$ Neil Walker, Loot Boxes Should Face Gambling Regulation, VentureBeat (Feb. 20, 2018), https://venturebeat.com/2018/02/20/loot-boxes-should-face-gamblingregulation/.
${ }^{33}$ Major League Baseball Props., Inc. v. Price, 105 F. Supp. 2d 46, 51 (E.D. N.Y. 2000). 
The same, however, cannot be said for a loot box. While a baseball card stands alone as its own compartmentalized product, a loot box only exists in the context of a greater whole. It exists, almost by definition, to modify the experience of an existing game that the player already possesses. If the player's copy of Battlefront II is lost, destroyed, or sold, then the money that the player invested in the accompanying loot boxes disappears with it. This dichotomy runs counter to the reasoning used by the court in Price and later posited by the ESRB - specifically, this refutes the notion that a gamer receives something of value when purchasing a loot box for a video game in the same way that he receives something of value when purchasing a booster pack of trading cards. While the player is technically receiving something of value, it is only when considered and appreciated in conjunction with another product. While this discrepancy does not dismantle the ESRB's argument entirely, it does dampen the persuasiveness of the comparison and highlights the novelty of the loot box dilemma.

Loot boxes are the cumulative effect of trend-driven evolution since the first time Mario snatched up a gold coin and triggered the addictive two-note jingle. Loot boxes represent a disheartening but altogether inevitable culmination of the gaming industry's worst impulses. Coupled with the addicting quality of the games themselves and the proclivity of players (particularly young ones) to become fixated on certain games, loot boxes are a dangerous step toward the "nursery casinos" that have so concerned representatives like Ranker and Lee and commentators like Martinelli and Schwartz. This issue is not as simple as anything so innocuous as a floating coin, but it draws from the same well. The ESRB's failure to address the issue represents a failure to protect impressionable gamers and inform responsible adults as to the potential hazards that these loot boxes and pay-to-win systems pose.

In the same sense that the ESRB issues warnings for other unsavory or inappropriate content (violence, language, and sexual themes), the organization owes a duty to potential buyers (adults and parents alike) to warn against hazardous or predatory gambling mechanics. Their failure to meet this duty by hiding behind a misplaced comparison to trading cards and a general unwillingness to prod the gaming industry's biggest cash cow represents a betrayal to the fundamental mission of their organization. By including loot box mechanics on their list of hazardous materials and issuing visible warnings to potential buyers, the ESRB can take a necessary first step towards remediating its mistake and curbing the spread of predatory business practices. Loot boxes stand at the center of this epidemic, and regulating this mechanic will increase awareness of the issue and encourage consumers (particularly parents) to take necessary precautions and inhibit the effect that loot boxes will have on their gaming experience and, more importantly, their wallets. 